ARTICLE

\title{
Probing electronic structure in berkelium and californium via an electron microscopy nanosampling approach
}

\author{
Alexander Müller ${ }^{1,2,6}$, Gauthier J.-P. Deblonde (10 3,4,6, Peter Ercius (10 1, Steven E. Zeltmann¹,2, \\ Rebecca J. Abergel (1) ${ }^{3,5 凶}$ \& Andrew M. Minor (1) $1,2 \bowtie$
}

Due to their rarity and radioactive nature, comparatively little is known about the actinides, particularly those with atomic numbers higher than that of plutonium, and their compounds. In this work, we describe how transmission electron microscopy can provide comprehensive, safe, and cost-effective characterization using only single nanogram amounts of highlyradioactive, solid compounds. Chlorides of the rare elements berkelium and californium are dropcast and then converted in situ to oxides using the electron beam. The f-band occupancies are probed using electron energy loss spectroscopy and an unexpectedly weak spinorbit-coupling is identified for berkelium. In contrast, californium follows a jj coupling scheme. These results have important implications for the chemistries of these elements and solidify the status of californium as a transitional element in the actinide series.

\footnotetext{
${ }^{1}$ National Center for Electron Microscopy, Molecular Foundry, Lawrence Berkeley National Laboratory, Berkeley, CA, USA. ${ }^{2}$ Department of Materials Science and Engineering, University of California, Berkeley, CA, USA. ${ }^{3}$ Chemical Sciences Division, Lawrence Berkeley National Laboratory, Berkeley, CA, USA.

${ }^{4}$ Glenn T. Seaborg Institute, Physical \& Life Sciences, Lawrence Livermore National Laboratory, Livermore, CA, USA. ${ }^{5}$ Department of Nuclear Engineering, University of California, Berkeley, CA, USA. ${ }^{6}$ These authors contributed equally: Alexander Müller, Gauthier J.-P. Deblonde. ${ }^{凶}$ email: rjabergel@lbl.gov; aminor@|bl.gov
} 
M any radioactive materials have unique properties and several of them play central roles in applications such as nuclear energy and cancer therapy ${ }^{1-5}$. However, the health hazards posed by radioactive elements have always been a major hurdle to their research. As a result, comparatively little is known about them and their chemistries. Within the actinide series from actinium (Ac) to lawrencium ( $\mathrm{Lr}$ ), of which all isotopes are radioactive, the vast majority of research has been on uranium (U), thorium (Th), neptunium (Np), and plutonium $(\mathrm{Pu})$. Further, of all described actinide-containing crystal structures ( 7000), only about $0.5 \%$ include elements with atomic numbers greater than that of $\mathrm{Pu}^{6}$. This imbalance can in part be explained by the relevance of the early actinides to nuclear power, space exploration, and nuclear weapons programs ${ }^{1-5}$, in part by the natural occurrence of thorium and uranium, on which much research has been focused. Another key factor is the extreme challenges introduced when working with the heavy actinides. Transplutonium elements are without exception synthetic elements and their production requires long irradiation campaigns in high neutron flux nuclear reactions, followed by arduous purification steps performed in hot cells and using remote handling techniques ${ }^{7}$. These processes are time-consuming, difficult, and therefore inherently expensive, yet yield only minute amounts of the targeted isotopes. The current global production capabilities are milligram amounts of berkelium-249 $\left({ }^{249} \mathrm{Bk}\right)$ and californium-249 $\left({ }^{249} \mathrm{Cf}\right)$, sub-microgram amounts of einsteinium$254\left({ }^{254} \mathrm{Es}\right)$, and picogram-scale quantities of fermium-257 $\left({ }^{257} \mathrm{Fm}\right)^{7}$. Furthermore, all transcurium elements except for Cf $\left({ }^{249} \mathrm{Cf}\right.$, half-life $\approx 351$ years $)$ that are currently available in appreciable quantities only have relatively short-lived isotopes, which rapidly decay and emit large amounts of radiation. This prevents stockpiling and increases the logistical burden even further. Considerable efforts have therefore gone into minimizing the amounts required for experiments ${ }^{8,9}$, but typical characterization techniques, such as X-ray diffraction and X-ray absorption spectroscopy still require microgram to milligram amounts per sample ${ }^{10,11}$. In the case of transplutonium elements, even such small amounts can be cost-prohibitive, pose significant health hazards and necessitate large administrative and experimental controls. Only a few institutions worldwide are set up to tackle these challenging requirements, and even then, experiments can still fail due to self-irradiation-induced artifacts ${ }^{12-14}$

In light of these challenges, the use of transmission electron microscopy (TEM), a technique allowing detection and investigation of even single atoms, seems like a promising approach ${ }^{15-20}$. Yet, we are only aware of two research groups that have used TEM to study transplutonium elements. In the 1970 's, a group at the Oak Ridge National Laboratory used TEM to solve crystal structures from polycrystalline diffraction patterns ${ }^{13,14,21}$ and in the $2000^{\text {'s, a }}$ group at Lawrence Livermore National Laboratory used electron energy loss spectroscopy (EELS) to investigate electronic structures $^{22-27}$. Neither group took advantage of the method's small detection limit but either worked with the maximum amounts available to them or started from a bulk sample, which was then prepared for TEM using traditional sample preparation methods ${ }^{23}$. Recent improvements have made TEM significantly more powerful and it can now even be used to investigate single atoms.

Here, we leverage these improved capabilities and complement them with a workflow that allows working with amounts as small as a single nanogram to explore crystal and electronic structures of $\mathrm{Bk}$ and $\mathrm{Cf}$ oxides. Both elements are exceedingly rare and expensive and their high radioactivity poses a serious health risk. Further, ${ }^{249} \mathrm{Bk}$ (half-life $\approx 330$ days) $\beta^{-}$-decays to ${ }^{249} \mathrm{Cf}$. Samples thereby self-contaminate and experiments benefit from a fast workflow and, when possible, processing of the samples close to the characterization facility. TEM provides a way to analyze morphology, crystal structure, and electronic structure in a safe, cost-effective, and rapid manner. EELS allows investigating the $\mathrm{f}$ band occupancies of Bk- and Cf-compounds and an unexpectedly weak spin-orbit coupling is determined for Bk.

\section{Results}

Sample preparation. TEM samples of actinide compounds were prepared by dropcasting aqueous acidic solutions of the chloride salts onto the carbon-film side of a standard TEM grid and letting the samples dry at room temperature and ambient pressure (Fig. 1 and Supplementary Fig. 1). Each drop had a volume smaller than $1 \mu \mathrm{l}$ and contained between 1 and $10 \mathrm{ng}$ of the actinide. As the liquid evaporated and the drop contracted, the concentration of the solute in the drop rose until it supersaturated and particles precipitated. In an idealized case, one would expect even contraction of the drop and, ultimately, material deposition in a tiny area of the TEM grid. In practice, contact-line-pinning was a dominant factor that caused uneven contraction of the drop and the precipitation of particles on geometrical features (Supplementary Fig. 1). Prior to working with rare, radioactive elements, we validated the preparation approach using drops containing $1 \mathrm{ng} \mathrm{SmCl}_{3}$, a non-radioactive surrogate with respect to ion size ${ }^{28}$ and solubility ${ }^{29}$. This yielded a carpet of evenly distributed, presumably hydrated, $\mathrm{SmCl}_{3}$ particles with well-defined facets (Supplementary Fig. 2).

We then moved on to experiments with the isotopes ${ }^{243} \mathrm{Am}$, ${ }^{248} \mathrm{Cm},{ }^{249} \mathrm{Bk}$, and ${ }^{249} \mathrm{Cf}$. In contrast to the preliminary experiments, none of the actinides deposited as neatly. We always found large, mostly electron-opaque deposits in the middle of the deposition area, which were identified as non-radioactive contaminants (Supplementary Figs. 3 and 4) using energy-dispersive X-ray spectroscopy (EDS) and EELS. The presence of these non-radioactive contaminants in actinide samples reveals another challenge unique to working with actinides: Their production is difficult and often focused on radiopurity rather than chemical purity. The detection of these previously overlooked, non-radioactive contaminants highlights the critical information that can be harvested via electron microscopy and could have large implications on the quality control of rare isotope production and nuclear forensics.

Structures of Cf- and Bk-containing particles. Cf-containing particles precipitated in a large area and in large amounts. These particles formed with a distinct, dumbbell-like morphology several micrometers in length and had a structure with pores along the long axis of the particle (Fig. 1e). Spectroscopic analysis confirmed the presence of $\mathrm{Cf}$ throughout the particle. It also indicated the presence of oxygen $(\mathrm{O})$ throughout the particle and of chlorine ( $\mathrm{Cl}$ ) at the surfaces (Fig. 1d). The particles were beamsensitive and it was not possible to accurately determine their chemical composition or crystal structure. However, strong $\mathrm{Cl}$, O, and Cf signals in EDS spectra suggested the formation of a hydrated chloride material (Supplementary Fig. 5a), which is in line with the use of a solution of californium chloride in hydrochloric acid. After extended electron-beam irradiation, a beamstable, polycrystalline compound formed in situ which had strong $\mathrm{Cf}$ and $\mathrm{O}$ signals in EDS and EELS, but no signs of $\mathrm{Cl}$ (Supplementary Fig. 5b). Electron diffraction proved challenging due to crystallite size and nearby contaminants, but the structure of the compound as seen in atomically-resolved high-angle annular dark-field (HAADF) STEM images was consistent with trigonal $\mathrm{Cf}_{2} \mathrm{O}_{3}$ (Fig. 2a) ${ }^{30} \cdot \mathrm{Cf}_{2} \mathrm{O}_{3}$ has in the past been prepared by heating $\mathrm{CfOCl}$ in water vapor or $\mathrm{O}_{2}{ }^{31}$, and we propose that the electronbeam irradiation ${ }^{32}$ had a similar effect.

In comparison, the sample prepared using the $\mathrm{Bk}(\mathrm{III})$ stock solution was significantly more contaminated to the point that 

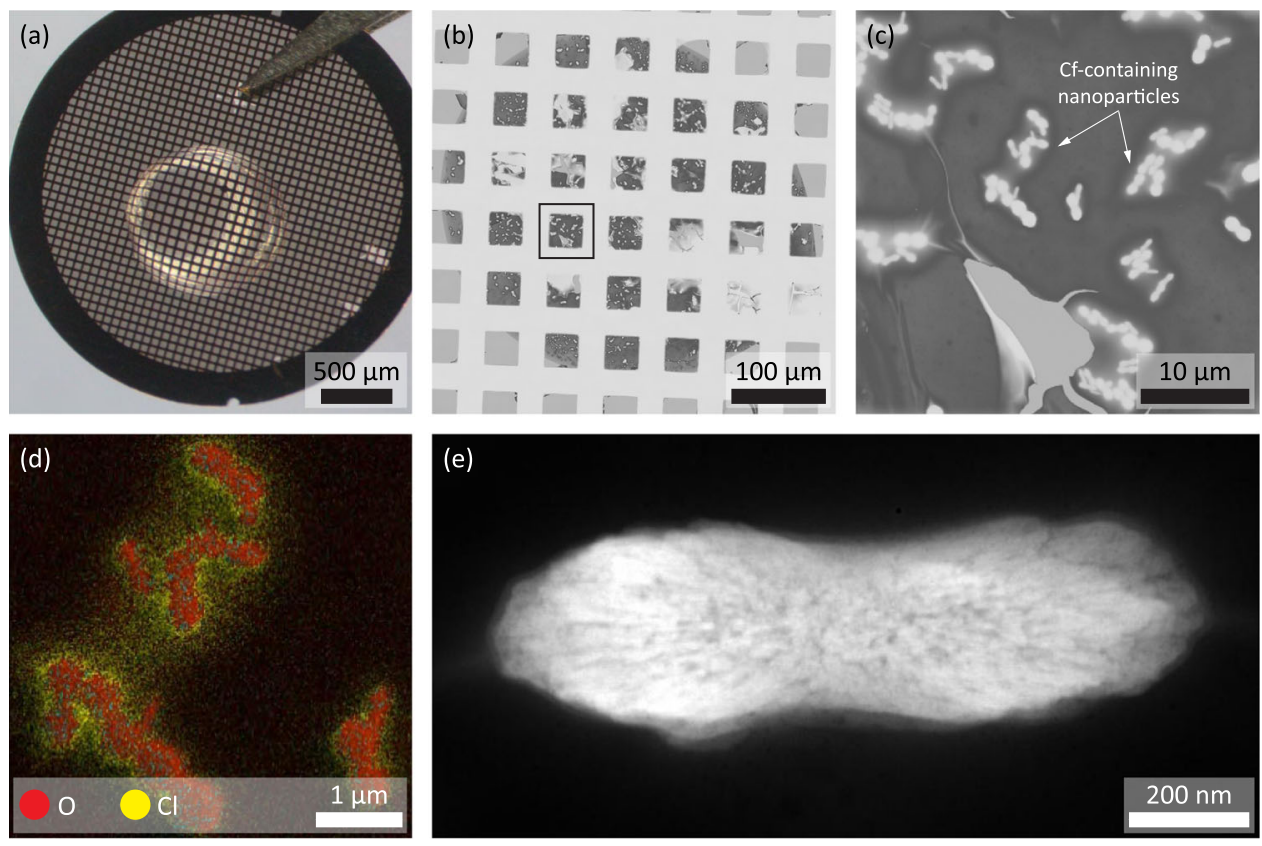

Fig. 1 Investigation of a dropcast Cf-containing sample. a Droplet of a solution containing Cf on a TEM grid. $\mathbf{b}$ The dried drop is imaged using the STEM detector of an SEM. A box marks the region shown in (c), in which individual particles can be seen. $\mathbf{d}$ EDS mapping, with $\mathrm{O}$ marked in red and $\mathrm{Cl}$ marked in yellow, confirms that the particles contain $\mathrm{O}$ and have $\mathrm{Cl}$-enriched surfaces. Individual spectra also confirm that the particles contain $\mathrm{Cf}$ (Supplementary Fig. 5). e HAADF-STEM image of individual particles shows the pores.
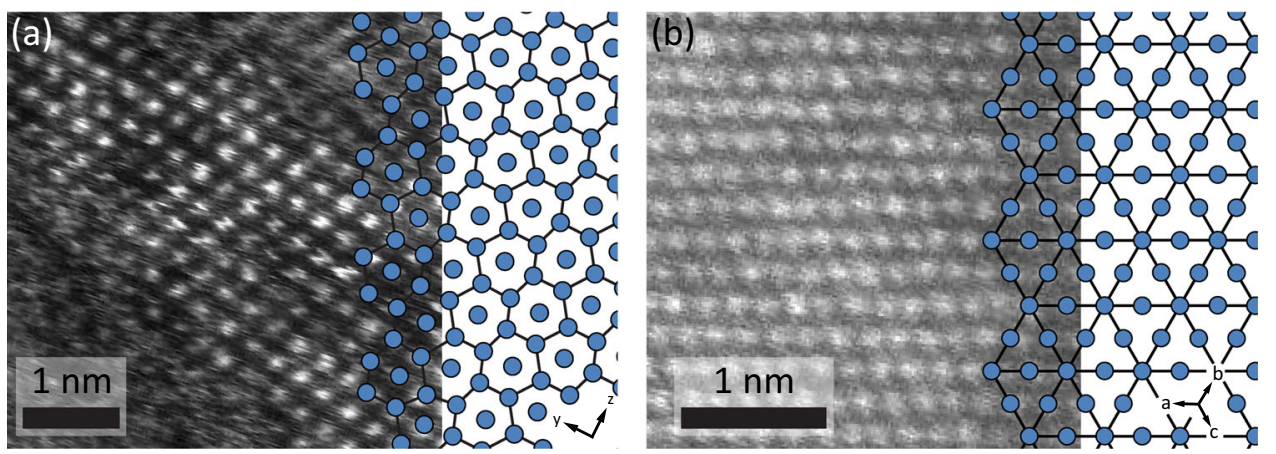

Fig. 2 Crystal structures of $\mathbf{C f}_{\mathbf{2}} \mathbf{O}_{\mathbf{3}}$ and $\mathbf{B k O}_{\mathbf{2}}$. a $\mathrm{Cf}_{2} \mathrm{O}_{3}$ viewed along the [100] lattice vector and partly overlaid schematic showing $\mathrm{Cf}$ columns. $\mathbf{b} \mathrm{BkO}$ viewed along the [111] lattice vector with $\mathrm{Bk}$ atoms shown in blue in the partly overlaid schematic. Please note that $\mathrm{O}$ atoms are not visible in the HAADFSTEM images and are therefore not shown in the schematic, either. In the schematics, atoms were connected by lines to accentuate structural motifs.

contaminants, particularly carbon (C), dominated. However, we were able to directly observe nanoparticles of cubic $\mathrm{BkO}_{2}$ (Fig. $2 \mathrm{~b}$ ) instead of needing extensive electron-beam irradiation ${ }^{33}$. We assume that the electron-beam-induced reaction happened too quickly to witness, as the direct formation of $\mathrm{BkO}_{2}$ from an aqueous solution is thermodynamically unfavorable. Nonetheless, the beaminduced formation of $\mathrm{BkO}_{2}$, with tetravalent $\mathrm{Bk}$, as opposed to $\mathrm{Bk}_{2} \mathrm{O}_{3}$, with trivalent $\mathrm{Bk}$, is in line with the relatively low redox potential of $\mathrm{Bk}(\mathrm{IV}) / \mathrm{Bk}(\mathrm{III})\left(+1.6 \mathrm{~V}\right.$ in $1 \mathrm{M} \mathrm{HClO}_{4}$ versus $\mathrm{NHE}$ ) compared to $\mathrm{Cf}(\mathrm{IV}) / \mathrm{Cf}(\mathrm{III})(+3.2 \mathrm{~V} \text { versus } \mathrm{NHE})^{34,35}$.

Electronic structures of Bk and Cf. Absorption edges of the actinides have mostly been observed using synchrotron-based Xray absorption spectroscopy (XAS), but only a handful of beamlines can accommodate radioactive materials ${ }^{36}$. Beside the logistical and administrative hurdles, even the microgram amounts required for XAS experiments often represent significant health hazards. EELS garners comparable data ${ }^{24}$, but excels at much lower energy ranges and requires significantly smaller sample amounts due to the increased beam-sample interactions of electrons compared to X-rays. Moore et al. successfully used EELS to study the $\mathrm{O}_{4,5}, \mathrm{~N}_{4,5}$, and $\mathrm{M}_{4,5}$ edges of several bulk actinide metals up to $\mathrm{Cm}^{1,25,27}$ and we now contribute the $\mathrm{O}_{4,5}, \mathrm{~N}_{4,5}$, and $\mathrm{M}_{4,5}$ edges of Bk and Cf (Fig. 3). Previously, only L edges of these two elements were measured using XAS. Initial measurements required hundreds of micrograms per sample ${ }^{37-39}$, although recent optimization reduced the required amount to $1-20 \mu \mathrm{g}$ per XAS sample ${ }^{40}$. This is, however, still about three orders of magnitude higher than in our experiments.

The $\mathrm{O}_{4,5}$ edges measure $5 \mathrm{~d} \rightarrow 5 \mathrm{f}$ transitions, but both the $\mathrm{O}_{4}$ and the $\mathrm{O}_{5}$ edges are contained in a broad edge commonly referred to as the giant resonance ${ }^{22}$. The onsets for Bk (Fig. 3a) and Cf (Fig. 3d) are at energy losses of 110 and $121 \mathrm{eV}$, respectively. As the ${ }^{249} \mathrm{BkO}_{2}$ sample also contained $\sim 15 \%{ }^{249} \mathrm{Cf}$ due to the former's radioactive decay, the measured $\mathrm{O}_{4,5}$ edge of $\mathrm{Bk}$ has a shoulder we ascribe to Cf. However, as the $\mathrm{O}_{4,5}$ edge of $\mathrm{Bk}$ is at a lower energy loss than that of Cf, its onset should be accurately determined. Both onsets also continue a trend established for the lighter actinides, 

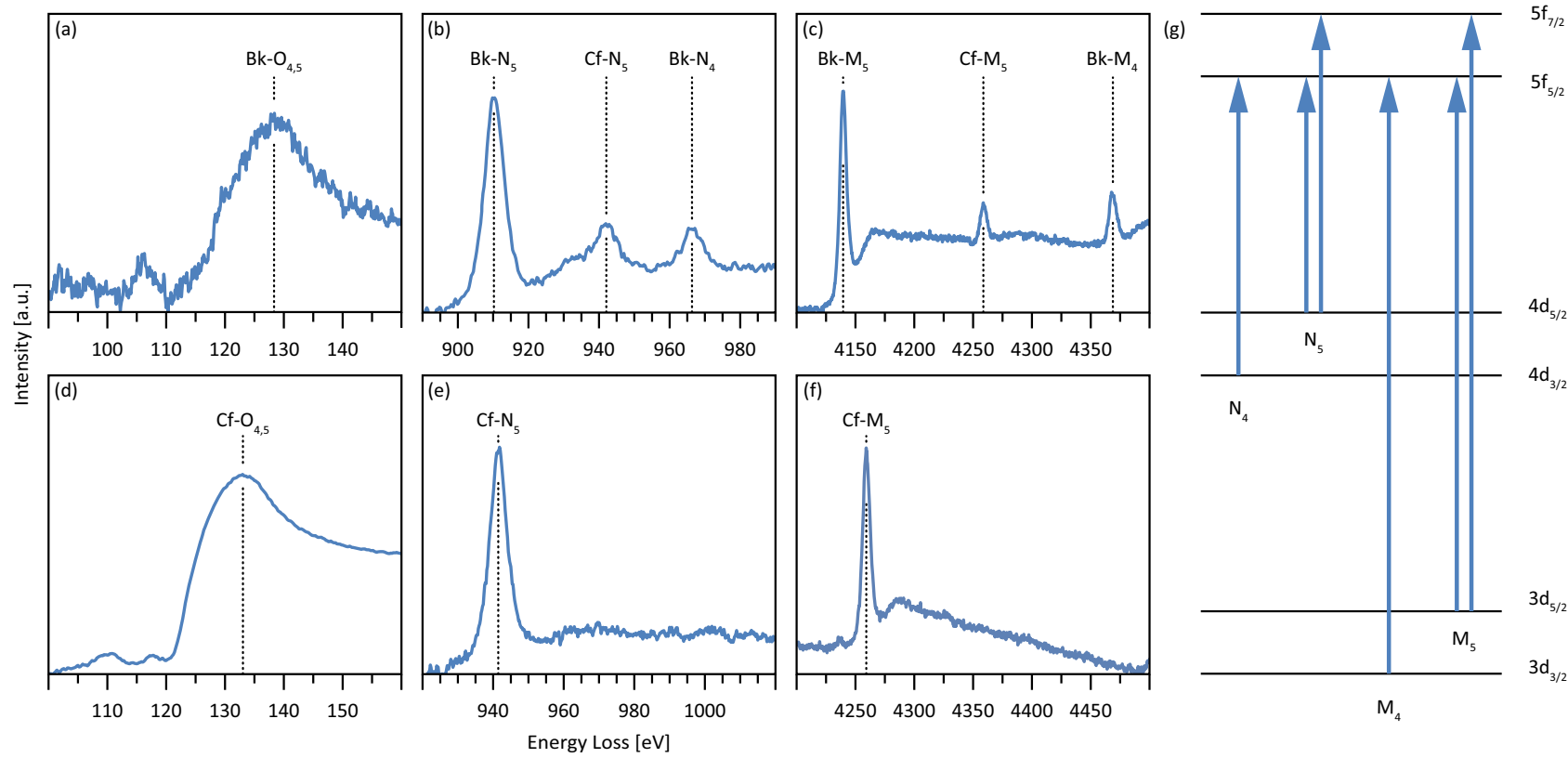

Fig. 3 Electronic structures of $\mathbf{B k}$ and Cf compounds. EELS spectra showing the $\mathbf{a} \mathrm{O}_{4,5}, \mathbf{b} \mathrm{N}_{4,5}$, and $\mathbf{c} \mathrm{M}_{4,5}$ edges of $B k$ and the $\mathbf{d} \mathrm{O}_{4,5}$, e $\mathrm{N}_{4,5}$, and $\mathbf{f} \mathrm{M}_{4,5}$ edges of Cf. The spectra of $\mathrm{Bk}$ have contributions from $\mathrm{Cf}$ that formed by radioactive decay. Please note that the scaling of the energy loss axis differs depending on whether $\mathrm{O}_{4,5}, \mathrm{~N}_{4,5}$, or $\mathrm{M}_{4,5}$ edges are shown. It is, however, consistent when comparing specific edges of the two elements. $\mathbf{g}$ Simple band diagram showing the transitions corresponding to the different white lines.

where the onset shifts by approximately $10 \mathrm{eV}$ from one actinide to the next ${ }^{27}$. Several peaks with a comparatively weak intensity precede the giant resonance peaks. These could have several causes, as described in the Supplementary Information, and we are therefore hesitant to assign physical meaning to them.

In contrast to the $\mathrm{O}_{4,5}$ edges, the $\mathrm{N}_{4,5}$ and $\mathrm{M}_{4,5}$ edges, which result from $4 \mathrm{~d} \rightarrow 5 \mathrm{f}$ and $3 \mathrm{~d} \rightarrow 5 \mathrm{f}$ transitions, respectively, have well-defined onsets and characteristic white lines (Fig. 3g). For Bk, the $\mathrm{N}_{4,5}$ (Fig. $3 \mathrm{~b}$ ) and $\mathrm{M}_{4,5}$ edges (Fig. 3c) have two welldefined white lines each. The $\mathrm{N}_{5}$ and the $\mathrm{N}_{4}$ edge have maxima at energy losses of 910 and $966 \mathrm{eV}$, respectively, and the $\mathrm{M}_{5}$ and the $\mathrm{M}_{4}$ edge have maxima at energy losses of 4140 and $4368 \mathrm{eV}$, respectively. In contrast, Cf only has one $\mathrm{N}_{5}$ white line (Fig. 3e) with a maximum at $942 \mathrm{eV}$ and one $\mathrm{M}_{5}$ white line (Fig. $3 \mathrm{f}$ ) with a maximum at $4259 \mathrm{eV}$ but is missing $\mathrm{N}_{4}$ and $\mathrm{M}_{4}$ white lines. Due to the decay of ${ }^{249} \mathrm{Bk}$ to ${ }^{249} \mathrm{Cf}$, the $\mathrm{N}_{5}$ and $\mathrm{M}_{5}$ white lines of $\mathrm{Cf}$ are also seen in the spectra of the Bk sample, but the edges are not expected to overlap with any features of the Bk edges. In spectra showing the M-edges (Fig. 3c, f), each of the white lines is followed by a second, rather broad peak at distances between 30 and $40 \mathrm{eV}$. These artifacts are likely due to multiple scattering processes involving plasmon excitations.

Of particular interest are the missing $\mathrm{N}_{4}$ and $\mathrm{M}_{4}$ edges of Cf, which normally result from the $4 \mathrm{~d}_{3 / 2} \rightarrow 5 \mathrm{f}_{5 / 2}$ and the $3 \mathrm{~d}_{3 / 2} \rightarrow 5 \mathrm{f}_{5 / 2}$ transitions, respectively (Fig. 3g). As electrons always transition from fully-filled d-bands, the intensities of these edges depend entirely on the occupancies of the $f_{5 / 2}$ band and the absence of the $\mathrm{N}_{4}$ and the $\mathrm{M}_{4}$ edge consequently indicates a fully-filled $\mathrm{f}_{5 / 2}$ band. Similar behavior has been observed for metallic $\mathrm{Cu}^{41,42}$. Our results are in line with f-band occupancies calculated from the formal oxidation states that follow from the previously determined chemical compositions ${ }^{43-47}$ : $\mathrm{Cf}(\mathrm{IV})$ with $5 \mathrm{f}^{8}$ in $\mathrm{CfO}_{2}$ and $\mathrm{Cf}(\mathrm{III})$ with $5 f^{9}$ in $\mathrm{Cf}_{2} \mathrm{O}_{3}$. These findings also continue a trend observed by Moore et al., who described a steady decrease in the intensity of the $\mathrm{N}_{4}$ peak of the actinides until Am and attributed it to a filling of the $5 f_{5 / 2}$ band under a $j j$ coupling scheme (spin-orbit coupling $>$ exchange interaction $)^{26}$. Moore et al. also described a break in this behavior for $\mathrm{Cm}$ : Metallic $\mathrm{Cm}$ has an $\mathrm{f}$-band occupancy of $5 \mathrm{f}^{7}$ and one would consequently expect a fully-filled $5 \mathrm{f}_{5 / 2}$ band and suppression of the $\mathrm{N}_{4}$ peak. Experiments contradicted this expectation, and they rationalized the unexpectedly intense $\mathrm{N}_{4}$ peak by a shift towards the LS coupling scheme (spin-orbit coupling $<$ exchange interaction), leading to electrons filling the $5 f_{5 / 2}$ as well as the $5 f_{7 / 2}$ band. We have confirmed their results with oxidized Am and $\mathrm{Cm}$ compounds prepared using our experimental approach (Supplementary Figs. 6 and 7), and extend their line of reasoning to our findings. Bk, which in our samples has an expected configuration of $5 \mathrm{f}^{\mathrm{\gamma}}\left(\mathrm{Bk}(\mathrm{IV})\right.$ in $\mathrm{BkO}_{2}$ ) and therefore could fill the $\mathrm{f}_{5 / 2}$ band under a $j j$ coupling scheme, behaves similarly to $\mathrm{Cm}$ and is shifted towards an LS coupling scheme, whereas Cf behaves like the early actinides and adheres to a $j j$ coupling scheme.

Experimental observations made in the 1960 's and $1970{ }^{\prime} \mathrm{s}^{4-47}$, and recently confirmed ${ }^{10,11,48-50}$, indicate that the transplutonium element series, unlike the analogous lanthanide series, is not monotonic. In particular, there is a growing body of evidence showing a pivot starting at $\mathrm{Bk}$ and $\mathrm{Cf}$. Previous observations were made via solution thermodynamics experiments (complex formation constants and liquid-liquid extraction behavior), magnetic measurements, density functional theory (DFT) calculations, and structure determinations (extended X-ray absorption fine structure (EXAFS) and single crystal diffraction) with notably shorter-thanexpected bond distances in the case of Cf compounds $s^{5,10,11,40,50}$.

Conclusion and outlook. In this work, we demonstrated the comprehensive analysis of actinide-containing, crystalline particles using amounts, and thereby radioactivity levels, several orders of magnitude lower than with commonly employed methods. By working with such small amounts, we successfully reduced administrative and engineering controls, accelerated workflows, limited the radiological exposure to workers and equipment, used non-dedicated equipment, reduced selfirradiation artifacts, decreased the costs associated with 
materials procurement and waste disposal, and managed to work with heavily contaminated, yet precious, samples. The comparative study of the f-band occupancies of Bk and Cf yielded an unexpected change in the strength of the spin- orbit coupling relative to the exchange interaction and thereby further bolsters the status of $\mathrm{Cf}$ as a transitional element within the actinide series. Our approach can be extended to other radioactive materials and allows not only complementing prior studies of the early actinides, but also opens an avenue for exploring the solid-state chemistry of elements such as einsteinium $\left({ }^{252} \mathrm{Es}\right.$ and ${ }^{254} \mathrm{Es}$, respective half-lives: 472 and 276 days), fermium $\left({ }^{257} \mathrm{Fm}\right.$, half-life: 100 days), and mendelevium ( ${ }^{258} \mathrm{Md}$, half-life: 51 days) for the first time.

\section{Methods}

Hazardous materials precautions. The four actinide isotopes investigated in this study were ${ }^{243} \mathrm{Am}\left(t_{1 / 2}=7388\right.$ years, specific activity $\left.=14.8 \mathrm{GBq} / \mathrm{g}\right),{ }^{248} \mathrm{Cm}\left(t_{1 / 2}=\right.$ $34,000$ years, specific activity $=0.16 \mathrm{GBq} / \mathrm{g}),{ }^{249} \mathrm{Bk}\left(t_{1 / 2}=330\right.$ days, specific activity $=$ $61 \mathrm{TBq} / \mathrm{g})$, and ${ }^{249} \mathrm{Cf}\left(t_{1 / 2}=352\right.$ years, specific activity $\left.=0.15 \mathrm{TBq} / \mathrm{g}\right)$. Further noteworthy is that ${ }^{243} \mathrm{Am}$ reaches a secular equilibrium with ${ }^{239} \mathrm{~Np}\left(t_{1 / 2}=2.356\right.$ days $)$ within $\sim 24$ days and that ${ }^{249} \mathrm{Bk}$ decays to ${ }^{249} \mathrm{Cf}$. All of these isotopes are highly radioactive, present serious health risks, and should be handled with caution.

Consequently, preparation of the stock solutions and dropcasting onto TEM grids took place in laboratories specifically dedicated to the safe handling of radioactive materials. Regular radiation protection standards were then followed for the transfer to the National Center for Electron Microscopy (NCEM).

As TEM samples were prepared with only $1-10 \mathrm{ng}$ of each isotope, the activities of the finished samples were rather low. For reference, $1 \mathrm{ng}$ of freshly purified ${ }^{243} \mathrm{Am}$ has an activity of about $30 \mathrm{~Bq}, 1 \mathrm{ng}$ of ${ }^{248} \mathrm{Cm}$ one of $1.2 \mathrm{~Bq}, 1 \mathrm{ng}$ of ${ }^{249} \mathrm{Cf}$ one of $151 \mathrm{~Bq}$, and $1 \mathrm{ng}$ of ${ }^{249} \mathrm{Bk}$ one of $59 \mathrm{kBq}$. The TEM samples are considered non-dispersible, and repeated tests indicated that even dropping a grid from a height of a few centimeters did not cause material to come loose. As the samples' radioactivity levels are several orders of magnitude below the applicable thresholds given in the Department of Energy (DOE) guideline 10 CFR 835, Appendix E, microscope rooms are not designated as "radioactive material areas" and the samples can be analyzed on non-dedicated electron microscopes. While these particular thresholds only apply to DOE labs, we assume that similar guidelines are in place at other institutions.

We nonetheless took a conservative approach and implemented several safety precautions at NCEM. All doors and work areas were labeled to indicate that radioactive materials were in use and that radiochemistry training was required to access them. The tables where the samples were mounted were covered with paper sheets to prevent contamination should a sample drop. Geiger counters (alpha and beta probes) were used to survey all work areas and all tools prior to and after working with samples. Each isotope had a dedicated set of tweezers to prevent cross-contamination. Further, we isolated the samples from the holders by using clips and washers dedicated to working with radioactive materials.

Sample preparation. Chemically purified $\mathrm{Am}, \mathrm{Cm}, \mathrm{Bk}$, and $\mathrm{Cf}$ salts from evaporated $\mathrm{HCl}$ solutions were purchased from Oak Ridge National Laboratory (ORNL). The ${ }^{248} \mathrm{Cm}$ starting material had an isotopic purity of $95.78 \%(4.12 \%$ ${ }^{246} \mathrm{Cm}, 0.06 \%{ }^{245} \mathrm{Cm}, 0.02 \%{ }^{244} \mathrm{Cm}$, and $0.02 \%{ }^{247} \mathrm{Cm}$ isotopic distribution by atom percentage). The ${ }^{249} \mathrm{Bk}$ starting material contained $<0.05 \mathrm{ppm}$ of ${ }^{252} \mathrm{Cf}$. Due to the radioactive decay of ${ }^{249} \mathrm{Bk}$ into ${ }^{249} \mathrm{Cf}$, the ${ }^{249} \mathrm{Bk} /{ }^{249} \mathrm{Cf}$-ratio at the time of the electron microscopy measurements (i.e., 65 days after the $\mathrm{Bk} / \mathrm{Cf}$ separation performed at ORNL) was 6.84 .

To prepare stock solutions for dropcasting, actinide chloride salts were dissolved in $0.1 \mathrm{M} \mathrm{HCl}$ (Standard, VWR Chemical BDH). The stock solutions were diluted with Milli-Q water to the $\mu \mathrm{M}$ level. TEM grids with ultrathin carbon films (Ted Pella Inc.) were held with dedicated negative-action tweezers and drops between 0.2 and $0.8 \mu \mathrm{L}$ in volume were deposited onto them and left to dry in air at room temperature. The evaporation of the drop took about $30 \mathrm{~min}$ and was observed using an optical microscope (Supplementary Fig. 1). Once dried, samples were packaged and safely transferred to the National Center for Electron Microscopy.

Scanning electron microscopy. A FEI Helios G4 UX was used to investigate the samples after receiving them at the microscope facility and to identify compounds of interest. All grids were mounted into a STEM sample holder reserved for radioactive materials. To acquire secondary electron images using the in-lens detector, the microscope was run in the immersion mode and at acceleration voltages between 1 and $5 \mathrm{kV}$. Further, STEM images were acquired using the HAADF-STEM detector at an acceleration voltage of $20 \mathrm{kV}$. The software MAPS was used to prepare large-scale, high-resolution images by sequentially acquiring thousands of images and stitching them. This large-scale image acquisition had the beneficial effect of immobilizing carbon compounds and minimizing carbon deposition during the TEM measurements. The SEM images were then analyzed manually to identify different components, whose chemistry was then probed by acquiring EDS maps using an acceleration voltage of $20 \mathrm{kV}$ and an EDAX Elect Octane Super spectrometer.

Transmission electron microscopy. Three transmission electron microscopes were used in this study, all operated at $300 \mathrm{kV}$. All experimental results were confirmed using several measurements.

Morphology and composition were mostly investigated using a FEI Titan or a FEI Titan Themis. The FEI Titan is equipped with a Gatan Ultrascan 1000 CMOS camera, a Gatan STEM detector, and a Bruker SuperX EDS detector. The imagecorrected FEI Titan Themis is equipped with a Ceta2 CMOS camera, two STEM detectors, and a Bruker SuperX EDS detector.

The TEAM 0.5 microscope, a double-corrected, monochromated FEI Titan was used to acquire high-resolution STEM images and EELS spectra. As we were not looking at fine features in the EELS spectra, an energy resolution of $0.9 \mathrm{eV}$ (no monochromation) was used. M-edges were acquired at extraction voltages of 4000 and $4050 \mathrm{eV}$ to prevent any overlap of the FEG extraction voltage effect with the $\mathrm{M}$-edges of $\mathrm{Bk}$ or $\mathrm{Cf}^{1,2}$. The TEAM 0.5 microscope is equipped with a special stage, a tilt-rotate design that allows full $360^{\circ}$-rotation range about both axes. This stage takes circular samples with a diameter of $1 \mathrm{~mm}$, and parts of the grids were punched out and then glued into rings. In this way, the TEM holder is never directly in contact with the radioactive samples.

\section{Data availability}

All relevant images and spectra analyzed during this study are included in the article and its supplementary information. Raw data is available from the corresponding authors on request.

Received: 25 September 2020; Accepted: 18 January 2021; Published online: 11 February 2021

\section{References}

1. Moore, K. T. \& Van der Laan, G. Nature of the $5 \mathrm{f}$ states in actinide metals. Rev. Mod. Phys. 81, 235-298 (2009).

2. McDevitt, M. R. et al. Feed-forward alpha particle radiotherapy ablates androgen receptor-addicted prostate cancer. Nat. Commun. 9, 1629 (2018).

3. Thiele, N. A., Woods, J. J. \& Wilson, J. J. Implementing f-block metal ions in medicine: tuning the size selectivity of expanded macrocycles. Inorg. Chem. 58, 10483-10500 (2019).

4. Natrajan, L. S., Swinburne, A. N., Andrews, M. B., Randall, S. \& Heath, S. L. Redox and environmentally relevant aspects of actinide(IV) coordination chemistry. Coord. Chem. Rev. 266-267, 171-193 (2014).

5. White, F. D., Dan, D. \& Albrecht-Schmitt, T. E. Contemporary chemistry of berkelium and californium. Chem. - A Eur. J. 25, 10251-10261 (2019).

6. Arnold, P. L., Dutkiewicz, M. S. \& Walter, O. Organometallic neptunium chemistry. Chem. Rev. 117, 11460-11475 (2017).

7. Roberto, J. B. et al. Actinide targets for the synthesis of super-heavy elements. Nucl. Phys. A 944, 99-116 (2015).

8. Young, J. P., Haire, R. G., Fellows, R. L. \& Peterson, J. R. Spectrophotometric studies of transcurium element halides and oxyhalides in the solid state. J. Radioanal. Chem. 43, 479-488 (1978).

9. Peterson, J. R. in Lanthanide and Actinide Chemistry and Spectroscopy 221-238 (1980).

10. Deblonde, G. J.-P. et al. Spectroscopic and computational characterization of diethylenetriaminepentaacetic acid/transplutonium chelates: evidencing heterogeneity in the heavy actinide(III) series. Angew. Chem. - Int. Ed. 57, 4521-4526 (2018)

11. Silver, M. A. et al. Characterization of berkelium(III) dipicolinate and borate compounds in solution and the solid state. Science 353, aaf3762 (2016).

12. Hecker, S. S. The magic of plutonium: 5 f electrons and phase instability. Metall. Mater. Trans. A 35, 2207-2222 (2004).

13. Baybarz, R. D., Haire, R. G. \& Fahey, J. A. On the californium oxide system. J. Inorg. Nucl. Chem. 34, 557-565 (1972).

14. Haire, R. \& Baybarz, R. Studies of einsteinium metal. J. Phys. 40, 101-102 (1979)

15. Krivanek, O. L. et al. Atom-by-atom structural and chemical analysis by annular dark-field electron microscopy. Nature 464, 571-574 (2010).

16. Ramasse, Q. M. et al. Probing the bonding and electronic structure of single atom dopants in graphene with electron energy loss spectroscopy. Nano Lett. 13, 4989-4995 (2013).

17. Ishikawa, R., Shibata, N., Taniguchi, T. \& Ikuhara, Y. Three-dimensional imaging of a single dopant in a crystal. Phys. Rev. Appl. 13, 034064 (2020).

18. Lovejoy, T. C. et al. Single atom identification by energy dispersive x-ray spectroscopy. Appl. Phys. Lett. 100, 154101 (2012). 
19. Hage, F. S., Radtke, G., Kepaptsoglou, D. M., Lazzeri, M. \& Ramasse, Q. M. Single-atom vibrational spectroscopy in the scanning transmission electron microscope. Science 367, 1124-1127 (2020).

20. Spurgeon, S. R. et al. Nanoscale oxygen defect gradients in $\mathrm{UO}_{2+\mathrm{x}}$ surfaces. Proc. Natl Acad. Sci. USA 116, 17181-17186 (2019).

21. Haire, R. \& Baybarz, R. D. Identification and analysis of einsteinium sesquioxide by electron diffraction. J. Inorg. Nucl. Chem. 35, 489-496 (1973).

22. Moore, K. T. \& Van Der Laan, G. Accurate labeling of the light-actinide O4,5 edges. Ultramicroscopy 107, 1201-1206 (2007).

23. Moore, K. T. X-ray and electron microscopy of actinide materials. Micron 41, 336-358 (2010).

24. Moore, K. T. et al. Electron-energy-loss spectroscopy and X-ray absorption spectroscopy as complementary probes for complex f-electron metals: cerium and plutonium. Philos. Mag. 84, 1039-1056 (2004).

25. Moore, K. T., Van Der Laan, G., Wall, M. A., Schwartz, A. J. \& Haire, R. G. Rampant changes in $5 f_{5 / 2}$ and $5 f_{7 / 2}$ filling across the light and middle actinide metals: EELS, many-electron atomic spectral calculations and spin-orbit sum rule. Phys. Rev. B 76, 073105 (2007).

26. Moore, K. T. et al. Emergence of strong exchange interaction in the actinide series: the driving force for magnetic stabilization of curium. Phys. Rev. Lett. 98, 236402 (2007).

27. Butterfield, M. T., Moore, K. T., Van Der Laan, G., Wall, M. A. \& Haire, R. G. Understanding the $\mathrm{O}_{4,5}$ edge structure of actinide metals: electron energy-loss spectroscopy and atomic spectral calculations of Th, U, Np, Pu, Am, and Cm. Phys. Rev. B 77, 113109 (2008).

28. Lundberg, D. \& Persson, I. The size of actinoid(III) ions-structural analysis vs. common misinterpretations. Coord. Chem. Rev. 318, 131-134 (2016).

29. Wilmarth, W. R., Young, J. P., Haire, R. G. \& Peterson, J. R. Spectrophotometric studies of californium(III) ions in selected lanthanide trihalide hosts. J. Less-Common Met. 143, 183-193 (1988).

30. Baybarz, R. D. High-temperature phases, crystal structures and the melting points for several of the transplutonium sesquioxides. J. Inorg. Nucl. Chem. 35, 4149-4158 (1973).

31. Copeland, J. C. \& Cunningham, B. B. Crystallography of the compounds of Cf -II crystal structure and lattice parameters of Cf oxychloride and Cf sesquioxide. J. Inorg. Nucl. Chem. 31, 733-740 (1969).

32. Egerton, R. F., Li, P. \& Malac, M. Radiation damage in the TEM and SEM. Micron 35, 399-409 (2004).

33. Peterson, J. R. \& Cunningham, B. B. Crystal structures and lattice parameters of the compounds of berkelium I. Berkelium dioxide and cubic berkelium sesquioxide. Inorg. Nucl. Chem. Lett. 3, 327-336 (1967).

34. Stokely, J. R., Baybarz, R. D. \& Peterson, J. R. The formal potential of the Bk (IV)-Bk(III) couple in several media. J. Inorg. Nucl. Chem. 34, 392-393 (1972).

35. Nugent, L. J., Baybarz, R. D., Burnett, J. L. \& Ryan, J. L. Electron-transfer and f-d absorption bands of some lanthanide and actinide complexes and the standard (II-III) oxidation potential for each member of the lanthanide and actinide series. J. Phys. Chem. 77, 1528-1539 (1973).

36. Shi, W.-Q. et al. Exploring actinide materials through synchrotron radiation techniques. Adv. Mater. 26, 7807-7848 (2014).

37. Antonio, M. R., Williams, C. W. \& Soderholm, L. Berkelium redox speciation. Radiochim. Acta 90, 851-856 (2002).

38. Revel, R. et al. First investigation on the L edges of the ${ }^{249} \mathrm{Cf}$ aquo ion by X-ray absorption spectroscopy. Inorg. Chem. 38, 4139-4141 (1999).

39. Galbis, E. et al. Solving the hydration structure of the heaviest actinide aqua ion known: the californium(III) case. Angew. Chem. 122, 3899-3903 (2010).

40. Kelley, M. P. et al. Bond covalency and oxidation state of actinide ions complexed with therapeutic chelating agent 3,4,3-Ll(1,2-HOPO). Inorg. Nucl. Chem. Lett. 57, 5352-5363 (2018).

41. Leapman, R. D., Grunes, L. A. \& Fejes, P. L. Study of the $\mathrm{L}_{23}$ edges in the $3 \mathrm{~d}$ transition metals and their oxides by electron-energy-loss spectroscopy with comparisons to theory. Phys. Rev. B 26, 614-635 (1982).

42. Pearson, D. H., Ahn, C. C. \& Fultz, B. White lines and d-electron occupancies for the 3d and 4d transition metals. Phys. Rev. B 47, 8471-8478 (1993).

43. Baybarz, R. D. Dissociation constants of the transplutonium element chelates of 1,2-diaminocyclohexanetetraacetic acid. J. Inorg. Nucl. Chem. 28, 1055-1061 (1966).

44. Baybarz, R. D. Dissociation constants of the transplutonium element chelates of diethylenetriaminepentaacetic acid (DTPA) and the application of DTPA chelates to solvent extraction separations of transplutonium elements from the lanthanide elements. J. Inorg. Nucl. Chem. 27, 1831-1839 (1965).

45. Baybarz, R. D. Separation of transplutonium elements by phosphonate extraction. Nucl. Sci. Eng. 17, 463-467 (1963).

46. Gavrilov, K. A., Gvzdz, E., Starý, J. \& Seng, W. T. Investigation of the solvent extraction of californium, fermium and mendelevium. Talanta 13, 471-476 (1966).
47. Hubert, S., Hussonnois, M., Brillard, L., Goby, G. \& Guillaumont, R. Determination simultanee de constantes de formation de complexes citrique de l'Americium, du Curium, du Californium, de l'Einsteinium et du fermium. J. Inorg. Nucl. Chem. 36, 2361-2366 (1974).

48. Drader, J. A., Luckey, M. \& Braley, J. C. Thermodynamic considerations of covalency in trivalent actinide-(poly) aminopolycarboxylate interactions. Solvent Extr. Ion-. Exch. 34, 114-125 (2016).

49. Kelley, M. P. et al. Revisiting complexation thermodynamics of transplutonium elements up to einsteinium. Chem. Commun. 54, 10578-10581 (2018)

50. Cary, S. K. et al. A series of dithiocarbamates for americium, curium, and californium. Dalt. Trans. 47, 14452-14461 (2018).

\section{Acknowledgements}

Work at the Molecular Foundry was supported by the U.S. Department of Energy (DOE), Office of Science, Office of Basic Energy Sciences under Contract No. DE-AC0205CH11231. This work was also supported by the DOE, Office of Science, Office of Basic Energy Sciences, Chemical Sciences, Geosciences, and Biosciences Division at LBNL, under contract DE-AC02-05CH11231. The ${ }^{249} \mathrm{Bk}$ and ${ }^{249} \mathrm{Cf}$ chloride starting materials were supplied by the Isotope Program within the Office of Nuclear Physics in the DOE's Office of Science. S.E.Z. was supported by STROBE, a Science and Technology Center sponsored by the National Science Foundation. The authors appreciate the support of Dahlia An, Melissa Mannion, and William Rowley in establishing risk-mitigating procedures at the National Center of Electron Microscopy (NCEM) at the Molecular Foundry.

\section{Author contributions}

This work combined two disciplines: actinide chemistry, represented by G.J.-P.D. and R.J.A., and electron microscopy, represented by A.M., P.E., S.E.Z. and A.M.M. This work would not have been possible without many discussions throughout which open questions in the field of actinide chemistry were combined with the capabilities offered by electron microscopy, thereby setting the scope of this manuscript. G.J.-P.D. prepared the samples and optimized the dropcasting process. A.M., P.E. and S.E.Z. performed the scanning electron microscopy and transmission electron microscopy experiments. A.M. analyzed the data. All authors contributed to the writing and editing of this manuscript.

\section{Competing interests}

The authors declare no competing interests.

\section{Additional information}

Supplementary information The online version contains supplementary material available at https://doi.org/10.1038/s41467-021-21189-1.

Correspondence and requests for materials should be addressed to R.J.A. or A.M.M.

Peer review information Nature Communications thanks Thomas E. Albrecht-Schmitt, Edgar Buck, and the other, anonymous, reviewer(s) for their contribution to the peer review of this work.

Reprints and permission information is available at http://www.nature.com/reprints

Publisher's note Springer Nature remains neutral with regard to jurisdictional claims in published maps and institutional affiliations.

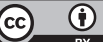

Open Access This article is licensed under a Creative Commons Attribution 4.0 International License, which permits use, sharing adaptation, distribution and reproduction in any medium or format, as long as you give appropriate credit to the original author(s) and the source, provide a link to the Creative Commons license, and indicate if changes were made. The images or other third party material in this article are included in the article's Creative Commons license, unless indicated otherwise in a credit line to the material. If material is not included in the article's Creative Commons license and your intended use is not permitted by statutory regulation or exceeds the permitted use, you will need to obtain permission directly from the copyright holder. To view a copy of this license, visit http://creativecommons.org/ licenses/by/4.0/.

This is a U.S. government work and not under copyright protection in the U.S.; foreign copyright protection may apply 2021 